\title{
A Novel Methodology in Modelling Dislocations for Analyzing Microstructures and Nanostructure Materials in Plastic Media
}

\author{
Hamed Farahmand
}

\begin{abstract}
Several methodologies have been proposed to model the effect of length scale parameters to improve constitutive equations for analyzing microstructures and nanostructure materials. Most of them are developed based on strain gradient theory. The main restriction is contributed to the large scale of imposed plastic deformation in comparison with implementation of length scale parameters. Also comparing to the scale of dislocation movement and hardening mechanisms, the plastic deformation in microstructures and nanostructure materials is sufficiently large that finite plasticity theory could be well justified. Therefore, the main intention of this paper is to develop strain gradient deformation with the corporation of finite plastic and dislocation theory as physically based attribution in constitutive equations. This procedure is accomplished with intrinsic length scale relation, which is dedicated to develop phenomenological of plasticity laws for microstructures in finite plasticity. Finally, the result of new theory gives for microstructures, and its predictable results are discussed for nanostructure materials.
\end{abstract}

Index Terms-Strain gradient plasticity; micromechanical modelling; dislocation theory; microstructures; nanostructure materials.

\section{INTRODUCTION}

A multiscale continuum is a bridge for a gap between classic continuum theories and micromechanical theories. The distinguished establishment of multiscale theory is defined on the basis of length scale parameters in the constitutive description. According to experimental data it is comprehended that the smaller size of material, the stronger is the response. In the conventional plasticity theory the dimension of microstructures and nanostructure deos not intervene in computations. First the result of indicating the size effects, explained by Aifantis [1]. Size effect is demonstrated in strain gradient as the spatial coefficients.

After that the strain gradient theory has been physically provoked by developing dislocation mechanics.

In the current work, first the basis concept of dislocation is described and then, relation between dislocation-based mechanics and continuum plasticity formulation is explored.

The mechanism-based strain gradient (MSG) plastic theory and the Taylor-based nonlocal theory (TNT) of plasticity i developed based on the Taylor-type flow stress as the starting point of this procedure afterwards the total dislocation densities in material is discussed which could be categorized into two different sections [2]:

H. Farahmand is with Department of mechanical Engineering, Young researcher club, Kerman Branch, Islamic Azad University, Kerman, Iran(email: hamed1256@yahoo.com; farahmand@iauk.ac.ir).
- Geometrically Necessary Dislocations (GNDs).

- Statistically Stored Dislocations (SSDs).

GNDs, which can be determined as crystallographic constrains leading to redundant crystal systems, were introduced by Nye [3] and Ashby [4] and developed by Arsenlis and Park [2]. GNDs are proportional to plastic strain gradient of material deformation.

SSDs, which could be considered as random trapping of dislocations, were explained by Ashby [4]. Moreover based on his approach, Kocks [5], [6], Esterin and Mecking [7], Kubin and Estrin [8], Bammann [9] and Beaudoin and Acharya [10] evolved equations for SSDs. SSDs is related to plastic strain of material deformation.

Although different mechanisms of dislocation are happened in plastic deformation phenomena such as cross slip, double cross slip of dislocation, pile up on grain boundaries and precipitate particles and arrange themselves in various types of cells or substructure networks but they can be well defined by dislocation densities.

According to dislocation theory, which developed classic plasticity theory for microstructures, a physically based strain gradient plasticity theory was evaluated for small deformation [11].

Significantly, the scale of plastic deformation in microstructures and nanostructure materials is sufficiently large in comparison with dislocation mechanisms and inner structural mechanisms for plastic deformation of these materials. Moreover, Yano et al. (2009)[12] conveyed that super plastic deformability is one of the properties of nanostructure materials which are produced in the way of severe plastic deformation (SPD). So, large strain could be occurred in these materials and finite plasticity deformation is an appropriate theory for modelling plastic deformation in microstructures and nanostructure materials. In order to expound the strain gradient, finite plastic deformation should be considered in the case of microstructures and nanostructure materials.

Relatively small number of papers has been devoted to gradient plasticity with finite deformation determination. Gurtin and Annand proposed the idea of large plasticity in strain gradient [13] and Cermelli and Gurtin proposed irrotational and spineless configuration [14]. Gao et al. [15] and Huang et al. [16] generalized finite deformation in the mechanism-based gradient plasticity theory. Chambon et al. [17] proposed a finite deformation phenomenological strain gradient plasticity which is analogous to fleck and Hutchinson [18]. Relying on finite deformation of strain gradient, Polizzotto commented the nonlocality. He explained that deformation in intermediate configuration should be stated based on strain gradient also featured the 
corrotational rate for strain gradient. He defined three dimensional tensor that could be stand for spin in intermediate space [19].

The layout of this paper is as follows: In section 2 kinematics of plastic deformation for finite plasticity theory is presented. In section 3 the modelling of dislocation is described. In section 4 formulation of finite plasticity theory of MSG theory is extended on the basis of dislocations theory then intrinsic length scale relation is used in the simulation of micro-torsion of thin wire. In section 5 numerical results are compared with experimental data.

\section{KinemATICS OF Plastic DeFormation}

Finite plastic deformation has been typically categorized into two sections where three configurations are defined through plastic deformation. Let a particle of continuous rigid body deforms in the case of $X^{0}=X^{0}(\bar{X}, t)$ where $\bar{X} \forall \bar{V}$ and $\bar{V}$ is an initial body volume at undeformed configuration which is designated as $\bar{B}$ at $\mathrm{t}=0 . X^{0}$ refers to intermediate configuration which can be proposed as an unstressed body. The rigid body rotation causes this transition. Thus the complementary part of this deformation is when the particle of the body undergo a deformation process $X=X\left(X^{0}, t^{0}\right)$ where $\mathrm{X}$ is refers to final location of the particle at deformed configuration $\mathrm{B}$ at $\mathrm{t}>0$. The deformation mapping of $X=X(\bar{X}, t)$ is illustrated in Fig. 1 .

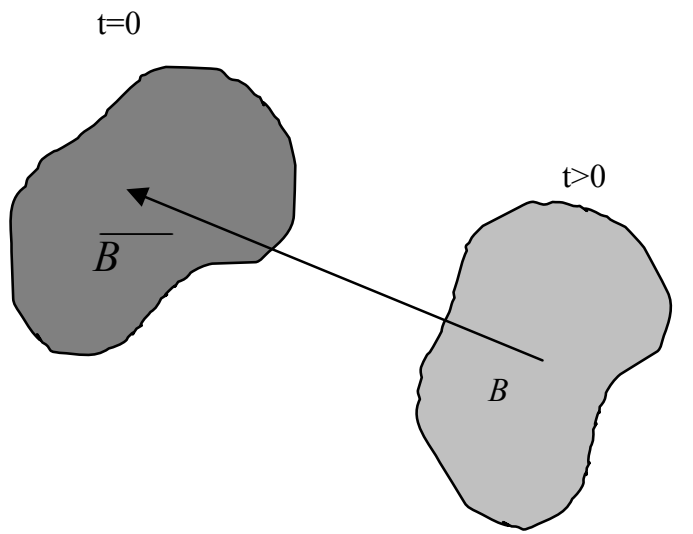

Fig. 1. Transition of a particle in different configurations.

According to conventional plastic deformation one of the most distinguish equation for modelling the stress-strain relationship is Levy-Mises criterion, which is nominated as

flow rule, can be described as $\stackrel{\circ}{\sigma}=\frac{2}{3} \frac{\dot{\sigma}^{e}}{D^{e^{p}}} D$ [20]. Where the effective stress is $\sigma^{e}=\sqrt{\frac{3}{2} \sigma_{i j} \sigma_{i j}}$, the effective rate of strain is $D^{e^{p}}=\sqrt{\frac{3}{2} D_{i j} D_{i j}}$ and ${ }^{\circ}$ is corrotational rate of stress [20].

When finite plasticity is used to promote the subject, intermediate configuration should be proposed. Hence appropriated corrotational rate of stress $\stackrel{\circ}{\sigma}$ should be considered. Furthermore substantial physical properties of plastic deformation should be taken into account for modelling the deformation of materials. So according to the scale of plastic deformation in comparison with inner structure and scale of dislocation mechanisms of microstructures and nanostructure materials and also the special role of dislocation mechanisms in plastic deformation of these materials, finite plasticity theory should be developed based on dislocation theory.

\section{DiSLOCATION MODELLING}

\section{A. Dislocation Description}

Plastic deformation is related to the motion of dislocations and also hardening is caused by interactions of dislocations. The impetus behind hardening phenomena is embedded the dislocation mechanisms. In microscopic point of view, a collection of dislocations is represented as the effect of dislocations. Therefore, dislocation density attribution is implemented for simulating of dislocation effects.

These arrangements act as obstacles to motion and thus providing the important mechanism of hardening. The continuum modelling of hardening mechanisms are explained on the basis of density of dislocations. The dislocation density concepts can be contributed into the continuum theories to alleviate the gap between conventional theory and micromechanical theory.

The Taylor hardening law, relating to the shear strength to and dislocation density, is the basis of (MSG) theory in plastic deformation. It is the best theory for explaining dislocation density in the plasticity.

The Taylor hardening law is explained as follows [20]

$$
\sigma^{e}=\sigma_{0}+m^{e} \alpha^{e} G b \sqrt{\rho_{i}}
$$

where $G$ is a shear modulus $b$ is the magnitude of Burgers vector and $\alpha$ is a material constant in the range of 0.1 to 0.5 related to crystals and $\rho i$ is the immobile or forest dislocation density. An average evaluation of Taylor factor (m) for polycrystal's material can be used in nonlocality constitutive equations ( $m=\sqrt{3}$ for isotropic solid and $m=3.08$ for FCC polycrystalline metal).

\section{B. Geometrically Necessary Dislocations and Statistically Stored Dislocations}

Dislocation density can be separated by two sections: GNDs and SSDs. The geometrically constrains of crystal lattice and obstacles which are indicated as strain gradient in equations, stand for GNDs and random trapping of dislocations during plastic deformation led to SSDs. Forest dislocations represents the total coupling of SSDs and GNDs.

Different combination of SSDs and GNDs can be deduced in the Taylor type description for total dislocation density. Mughrabi (2001) [21] concluded that the simple superposition of GNDs and SSDs is not well founded but, Abu Al-Rub and Voyiadjis (2005) [22],[23] presented different forms of GNDs and SSDs combination depending on the measuring of $\left(\left(\varepsilon^{p}\right)^{\gamma_{1}}+\left(\ell \partial \varepsilon^{p} / \partial x\right)^{\gamma_{2}}\right)^{1 / \gamma_{3}}$, where $\partial \varepsilon^{p} / \partial x$ is related to GNDs and $\varepsilon^{p}$ is referred to SSDs, 
also $\gamma_{1}, \gamma_{2}, \gamma_{3}$ are constant and $\ell$ is the length scale parameter.

The form of combination in this work is explained as

$$
\dot{\rho}_{i}=\dot{\rho}_{G}+\dot{\rho}_{S}
$$

where $\rho_{G}$ and $\rho_{S}$ represent GNDs and SSDs respectively and $\rho_{i}$ refers to total density. The total density should be emphatically supported the continuity of the crystal lattic.

The GNDs density simultaneously can not be created or annihilated as SSDs but it results from reaction of other regions existing GNDs, and enhenced to an effective plastic strain ( $\left.\dot{\varepsilon}^{p}\right)$ in small plasticity. Thus, based on Arsenlis and Park (1999)[2], Gao et al. (1999)[24], Huang et al. (2000) [16] and Hutchinson (2000) [25], GNDs is expressed as follows

$$
\dot{\rho}_{G}=\frac{\bar{r}}{b} \frac{\partial \gamma^{p}}{\partial x}
$$

where $\bar{r}$ is Nye factor and $\mathrm{b}$ is the magnitude of Burgers vector.

It was explained by Bamman and Aifantis (1982) [1] that increment in plastic strain tensor in microscopic plasticity theory $\left(\dot{\varepsilon}_{i j}^{p}\right)$ could be replaced by an effective plastic shear strain $\left(\dot{\gamma}^{p}\right)$ as follows

$$
\dot{\varepsilon}_{i j}^{p}=\dot{\gamma}^{p} m_{i j}
$$

where mij is symmetric Schmid's orientation tensor.

This equation can be rewritten as

$$
\dot{\gamma}^{p}=m \dot{\varepsilon}^{p}
$$

where $\dot{\mathcal{E}}^{p}$ is effective plastic strain, $\dot{\gamma}^{p}$ is effective plastic shear strain and $\mathrm{m}$ is the average the Taylor factor which is explained in Eq (5), Substituting in Eq (3) leads to [2]:

$$
\dot{\rho}_{G}=\frac{m \bar{r}}{b} \frac{\partial \varepsilon^{p}}{\partial x}
$$

In this work, GNDs modeling is generalized in finite plasticity as

$$
\dot{\rho}_{G}=\frac{m \bar{r}}{b}\left(\nabla \otimes D^{e^{p}}\right)
$$

where $D^{e^{p}}$ is effective rate of plastic strain.

In Eq (7) the term $\left(\nabla \otimes D^{e^{p}}\right)$ can be stated as tantamount spin in intermediate configuration and emphatically proposed in the directions, where the size of materials minimised. Therefore, Eq (7) for micro-torsion of thin wire where the radius of the wire is minimised, is changed to

$$
\dot{\rho}_{G}=\frac{m \bar{r}}{b} \frac{\partial D^{e^{p}}}{\partial r}
$$

In the case of calculation SSDs, the strain gradient is eliminated and only the flow stress depending on the plastic strain mechanism should be taken into account and it is proportional to $\varepsilon_{p}^{e}$ and $\left(\varepsilon_{p}^{e}\right)^{2}$. In regarding to Arsenlis and Park (1999) [2], the SSDs hardening mechanism is independent of GNDs mechanism. Hence, Kubin's model for hardening material of SSDs density is described as [8]

$$
\dot{\rho}_{S}=\left(\frac{k_{1}}{b} \sqrt{\rho_{S}}-K_{2} \rho_{S}\right) \dot{\gamma}^{p}
$$

where $\mathrm{k} 1$ and $\mathrm{k} 2$ are constant, $k_{1} \sqrt{\rho_{s}}$ represents immobilization and annihilation and $k_{2} \rho_{s}$ represents dynamic recovery.

Considering the relation between the effective plastic shear strain and effective plastic strain as $\dot{\gamma}^{p}=m \dot{\varepsilon}^{p}$, Eq (9) can be led to

$$
\dot{\rho}_{S}=m\left(\frac{k_{1}}{b} \sqrt{\rho_{s}}-k_{2} \rho_{s}\right) \varepsilon^{p}
$$

Furthermore, according to Kubin et al. (2002) [26], Kubin's model of dislocation can be generalized in finite plasticity. Hence an effective rate of plastic strain $D^{e^{p}}$ can be appropriately used in Kubin's model as follows

$$
\dot{\rho}_{S}=m\left(\frac{k_{1}}{b} \sqrt{\rho_{s}}-k_{2} \rho_{s}\right) D^{e^{p}}
$$

So the two categories of dislocation density which are considered by an effective rate of plastic strain are utilized to promote finite classic plasticity theory.

\section{THEORY FORMULATION}

\section{A. Dislocation Modelling in Constitutive Equations}

In this section, the dislocation mechanisms are modelled by finite plasticity theory.

Differentiating of the flow stress can be explained as (Voyiadjis, 2005) [22].

$$
\dot{\sigma}^{e}=\frac{\partial \sigma}{\partial \rho_{G}} \dot{\rho}_{G}+\frac{\partial \sigma}{\partial \rho_{S}} \dot{\rho}_{S}
$$

where $\rho_{S}$ is SSDs density and $\rho_{G}$ is GNDs density and also $\dot{\rho}_{i}=\dot{\rho}_{S}+\dot{\rho}_{G}$

$$
\dot{\sigma}^{e}=\frac{\partial \sigma^{e}}{\partial \rho_{i}}\left[\left(\frac{k_{1}}{b} \sqrt{\rho_{s}}-k_{2} \rho_{s}\right) m D^{e^{p}}+\frac{m \bar{r}}{b} \frac{\partial D^{e^{p}}}{\partial r}\right]
$$


Substitution of Eq (8) and Eq (11) in Eq (12) obtains Eliminating gradient terms in Taylor theory yields

$$
\sigma_{S}=\sigma_{0}+m G b \alpha \sqrt{\rho_{S}}
$$

In the case of average declaration, two useful equations are as follows

$$
\begin{gathered}
\rho_{i}=\left(\frac{\sigma-\sigma_{0}}{m G b \alpha}\right)^{2} \\
\rho_{S}=\left(\frac{\sigma_{S}-\sigma_{0}}{m G b \alpha}\right)^{2}
\end{gathered}
$$

Substituting Eq (15) in Eq (13) leads to

$$
\dot{\sigma}^{e}=\frac{(m \alpha G b)^{2}}{2\left(\sigma-\sigma_{0}\right)}\left[\left(\frac{k_{1}}{b} \sqrt{\rho_{s}}-k_{2} \rho_{s}\right) m D^{e^{p}}+\frac{m \bar{r}}{b} \frac{\partial D^{e^{p}}}{\partial r}\right]
$$

Following equation can be obtained by substituting Eq (16) in $\mathrm{Eq}(17)$.

$$
\dot{\sigma}^{e}=\frac{m^{3} \alpha^{2} G^{2} b \bar{r}}{2\left(\sigma^{e}-\sigma_{0}\right)}\left[\left(\frac{\partial D^{e^{p}}}{\partial r}\right)\right]+\frac{\sigma^{e}-\sigma_{0}}{\sigma^{e}-\sigma_{0}}\left[\left(\frac{m^{2} \alpha G k_{1}}{2}-\frac{k_{2} m}{2}\left(\sigma^{e}-\sigma_{0}\right)\right) D^{e^{p}}\right]
$$
to

In the absence of gradient rate of strain, Eq (18) is reduced

$$
\dot{\sigma}^{e} S=\frac{1}{2}\left[m^{2} \alpha G k_{1}-k_{2} m\left(\sigma_{S}-\sigma_{0}\right)\right] D^{e^{p}}
$$

For calculating the coefficients $k_{1}$ and $k_{2}$ the coefficient $h$ (the strain rate hardening) should be introduced as

$$
h=\frac{d \dot{\sigma}}{d D}
$$

Initial condition of finite plastic deformation equals to the initial condition of small deformation theory So, for initial condition of new plastic deformation of microstructures is defined as

$$
h_{0}=\frac{d \sigma_{s}}{d \varepsilon}
$$

Therefore $k_{1}$ and $k_{2}$ can be expressed as follows

$$
k_{1}=\frac{2 h_{0}}{m^{2} G \alpha \Omega} \quad, \quad k_{2}=\frac{2 h_{0}}{m\left(\sigma_{S a t}-\sigma_{0}\right)}
$$

According to the suggestion of Kubin and Estrin (1990) [8],
$\Omega$ can be neglected $(\Omega=1)$ and $\sigma_{\text {sat }}$ is saturated stress and equals $\sigma_{s}$ in the initial where no gradient terms exist.

Substitution $k_{l}$ and $k_{2}$ in Eq (19) gives

$$
\dot{\sigma}_{S}=h_{0}\left(\frac{\sigma_{s a t}-\sigma_{s}}{\sigma_{s a t}-\sigma_{0}}\right) D^{e^{p}}
$$

\section{B. Modelling of the Finite Strain Gradient Plasticity Theory}

In this work, the plastic deformation has been developed by the assumption of the classic plasticity and the dislocation mechanisms. Also dislocation theories for microstructures and nanostructure materials are developed by finite plasticity deformation theory.

In accordance with conventional plasticity, flow rule proposing the relation between stress and strain can be explained for microstructures as follows [20]

$$
\stackrel{\circ}{\sigma}^{J}=\frac{2}{3} \frac{\dot{\sigma}^{e}}{D^{e}} D
$$

where $\dot{\sigma}^{e}$ is an effective stress for microstructures and the evolving of the yield surface is directly related to dislocation theory, corresponding to $\dot{\sigma}^{e}$. So Eq (24) is developed by dislocation theory and Jaumann corrotational stress rate for microstructures.

Jaumann corrotational stress rate is defined as follows [20]

$$
\stackrel{\circ}{\sigma}^{J}=\dot{\sigma}-W \sigma+\sigma W
$$

Although Jaumann corrotational rate is exhibited oscillation behaviour, it can appropriately predict the form of plastic deformation for microstructures in experimental test. Therefore, on the basis of classic plasticity and plastic deformation, deformation gradient tensor for torsion is expressed as (Hwang et al., 2003) [27]

$$
F=\left[\begin{array}{lll}
\frac{d r}{d R} & 0 & 0 \\
0 & \frac{r}{R} & k r \\
0 & 0 & \psi
\end{array}\right]
$$

And velocity gradient tensor is defined as

$$
L=\dot{F} F^{-1}=\left[\begin{array}{ccccc}
\frac{\dot{\alpha}}{\alpha} & 0 & & 0 & \\
0 & & \frac{\dot{r}}{r} & & \dot{k} r \\
0 & 0 & \frac{\dot{\psi}}{\psi}
\end{array}\right]
$$


where $\alpha=\frac{d r}{d R}$.

So that symmetric part of $L$ which is the rate of strain is calculated as follows

$$
D=\left[\begin{array}{cccc}
\frac{\dot{\alpha}}{\alpha} & 0 & & 0 \\
0 & & \frac{\dot{r}}{r} & \frac{1}{2} \dot{k} r \\
0 & & \frac{1}{2} \dot{k} r & \frac{\dot{\psi}}{\psi}
\end{array}\right]
$$

Asymmetric part of $L$ which is material spin is proposed as

$$
w=\left[\begin{array}{ccc}
0 & 0 & 0 \\
0 & 0 & \frac{1}{2} k r \\
0 & -\frac{1}{2} k r & 0
\end{array}\right]
$$

So the effective rate of strain can be calculated as

$$
D^{e^{p}}=\sqrt{\frac{2}{3} D_{i j} D_{i j}}=\sqrt{\frac{2}{3}\left[\frac{\dot{\alpha}}{\alpha}\right]^{2}+\left[\frac{\dot{r}}{r}\right]^{2}+2\left[\frac{1}{2} \frac{\dot{k} r}{\psi}\right]^{2}+\left[\frac{\dot{\psi}}{\psi}\right]^{2}}
$$

where, based on Huang et al. (2003) [27], $\psi$ and $\alpha$ are respectively interrelated to the Axial strain effect and experimental test. It is assumed that $\psi$ and $\alpha$ are constant through the time stepping. Thus, the effective rate of strain is expressed as

$$
D^{e^{p}}=\sqrt{\left[\frac{\dot{r}}{r}\right]^{2}+2\left[\frac{1}{2} \dot{k} r\right]^{2}}
$$

Although Jaumann corrotational rate is exhibited oscillation behaviour, it can be appropriately predicted the form of plastic deformation for microstructures and nanostructure materials in experimental test so, Jaumann corrotational rate of stress is derived as follows

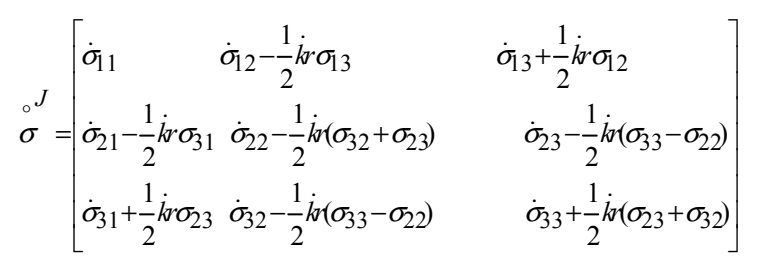

If it is defined two new parameters as follows

$$
\begin{gathered}
A_{1}=\frac{m^{3} \alpha^{2} G^{2} b^{2}}{2}\left(\frac{k_{1}}{b} \sqrt{\rho_{s}}-k_{2} \rho_{s}\right) \\
A_{2}=m^{3} \alpha^{2} G^{2} \bar{r} b
\end{gathered}
$$

The flow rule is simplified to calculate $\sigma_{32}$ as following form

$$
\frac{\partial^{2}\left(\frac{\sigma_{32}}{\sigma_{e}}\right)}{\partial k^{2}}-\left(\frac{\partial r}{r}\right) \frac{\partial\left(\frac{\sigma_{32}}{\sigma_{e}}\right)}{\partial k}+\frac{r^{2} \sigma_{32}}{2}=\left(\frac{\partial r}{3}\right) p_{1}-\frac{A_{2} p_{2}}{3 r}\left(\frac{\partial r}{\partial k}\right) p_{2}+\frac{8 A_{2} T}{9 r^{7}}
$$

where $\mathrm{P} 1$ and $\mathrm{P} 2$ and $\mathrm{T}$ are considered as follows

$$
\begin{gathered}
T=6 r^{2}\left(\frac{\left.\left.\frac{\partial r}{\partial k}\right)^{3}, \quad A_{1}+\frac{A_{2}}{r}\left(p_{2}\right)\right)}{p_{2}}=\frac{\left.\left(-\frac{\partial r}{\partial k}\right)^{2}+\frac{r^{2}}{2}\right)}{\left.\left(\frac{\partial r}{\partial k}\right)^{2}+\frac{r^{2}}{2}\right)}\right.
\end{gathered}
$$

\section{NUMERICAL ANALYSIS}

Initial condition is extracted, in accordance with the experimental data. Also $\rho_{S}$ can be calculated as (Voyadjis and Abu Al Rub, 2005) [22]

$$
\rho_{s}=\left[\frac{\sigma_{r e f}}{m \alpha G b}\left(1-\exp \left(\frac{-h_{0}}{\sigma_{r e f}} \varepsilon^{e^{p}}\right)\right]^{2}\right.
$$

where $\varepsilon^{e^{p}}$ is the effective plastic strain in small deformation and $\sigma_{r e f}=\sigma_{\text {sat }}-\sigma_{0}$. Refering to Fig. 2, it can be illustrated that in finite deformation, SSDs can be treated as a constant value $\left(\rho_{S}=4200\right)$.

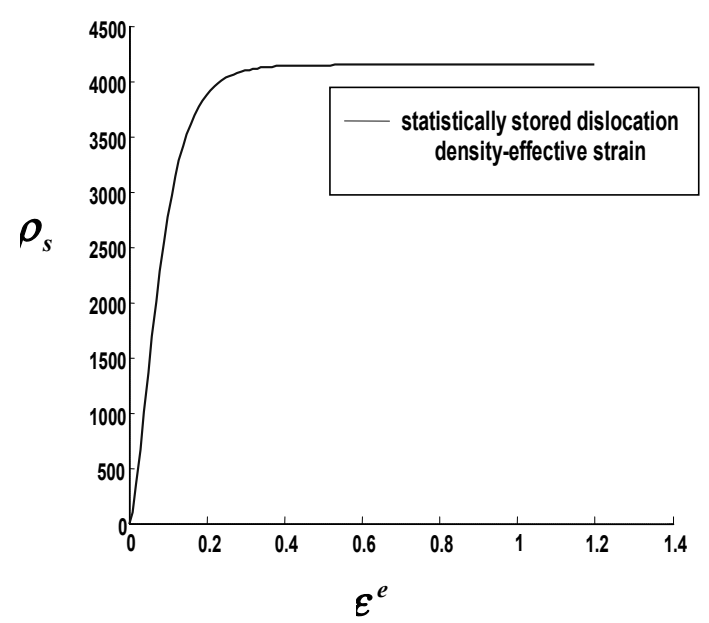

Fig. 2. Statistically stored dislocation density versus effective strain diagram according toVoyadjis results.

The constant value of $\mathrm{Eq} \mathrm{(34)} \mathrm{is} \mathrm{determined} \mathrm{for} \mathrm{the}$ micro-torsion test of thin Copper wires which are used in 
experimental test of Fleck et al. (1994) [28]. The experimental results are fitted with $\sigma_{\text {ref }}=226 \mathrm{MPa}, \mathrm{G}=44$ GPa and $\sigma_{0}=0 \mathrm{MPa}$. The Hall-Petch behaviour of $\sigma_{0}$ is not significant in these experiments as reported by Fleck et al. (1994) [28]. The microstructure parameters are $\mathrm{m}=3.08, \bar{r}$ $=1.93, \mathrm{~b}=0.255 \mathrm{~nm}, \mathrm{~h} 0=11500$, and $\alpha=0.1$. In this study, supposing $\frac{\partial r}{\partial k}=0.002$, provides reasonable agreement between experimental and new simulation data .So, Fig. 3a observes the relation between $\sigma_{32}$ and $\mathrm{k}$ (torsion parameter) for torsion of thin Copper wire with diameter of $2 \mathrm{a}=12 \mu \mathrm{m}$ ( $\sigma_{32}$ is computed in Eq $(20)$ ).

In accordance with Fig. 3a and Fig. 3b, it is deduced that the fluctuation of experimental stress for microstructures and variation form of stress, imposed by high pressure torsion (HPT) for nanostructure materials could be predicted by new finite plasticity model of (MSG) theory. Comparing Fleck et al. (1994) [28] experimental data with new finite plasticity model of (MSG), the relation between $\frac{\sigma_{32}}{\sigma_{e}}$ (stress parameter) and $\frac{Q}{a^{3}}$ (disturbance parameter) should be stated. In finite plasticity this relation can not be calculated as exact form so, the general form of this relation is explained as

$$
\frac{\sigma_{32}}{\sigma_{e}}=f\left(\frac{Q}{a^{3}}\right)
$$

where $\mathrm{Q}$ is the torque-twist and $a$ is radius of thin copper wire.

For linear elastic deformation of materials, Eq (37) is defined linear proportion but it should be suggested nonlinear form for finite plastic deformation. Hence, a kind of assuming parameters' relation for deriving more accurate data can be given as follows

$$
\left.\frac{\sigma_{32}}{\sigma_{e}}=\frac{556872}{a^{8}\left(A_{2}\right)} \exp \left(\frac{780 A_{1}\left(a^{2}\right)}{A_{2}\left(P_{1}\right)\left(\sigma_{r e f}\right)^{2}}\right)\left(\frac{Q}{a^{3}}\right)\right)
$$

This equation is considered to compare the present (MSG) of finite plastic deformation model's results with micro-torsion test of Fleck et al. (1994) [28] for thin Copper wires, which have different values of diameters $(2 \mathrm{a}=12,15$, $20 \mu \mathrm{m})$. The resulting data evaluation from $\mathrm{Eq}$ (38) is depicted in Fig. 4.

According to Fig. 4, Fig. 5 and Fig. 6 the results of Eq (38) significantly can be tracked the experimental data even for the thinner wire $(2 \mathrm{a}=12)$. Moreover, the results of New model is compared with Voyadjis et.al, Aifantis et.al and Gao et.al works in these figures, and the accuracy of New methodology is evaluated. As it is illustrated, more agreements between experimental data of Fleck et al. (1994) [28] and new model of (MSG) theory for finite plastic deformation is predicted on the basis of dislocation mechanisms.

The relation between stress and disturbance parameter is developed in nonlinear form. Also, radius of thin wire and different parameters, which are described in evaluation of stress, are taken corporate in this relation. Thus, the length scale is declared intrinsically in the form of relation in $\mathrm{Eq}$ (38). Hence, in this work the length scale parameter is replaced by the length scale relation and new finite plasticity theory of (MSG) and length scale relation observe better data for large value of torsion parameter $(\mathrm{k})$.

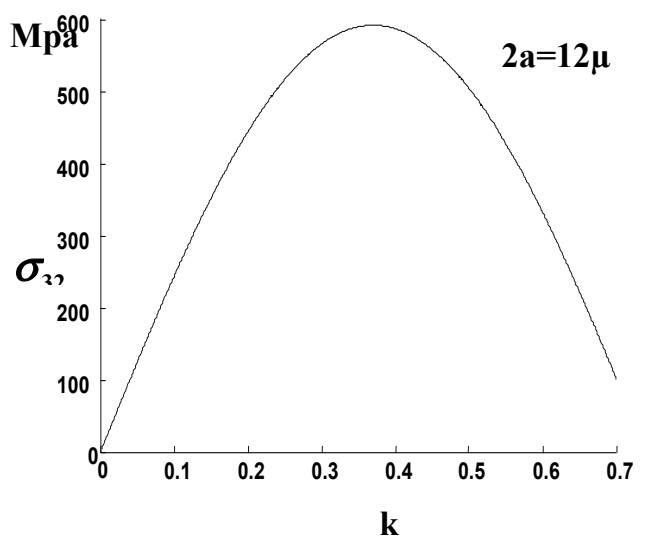

(a)

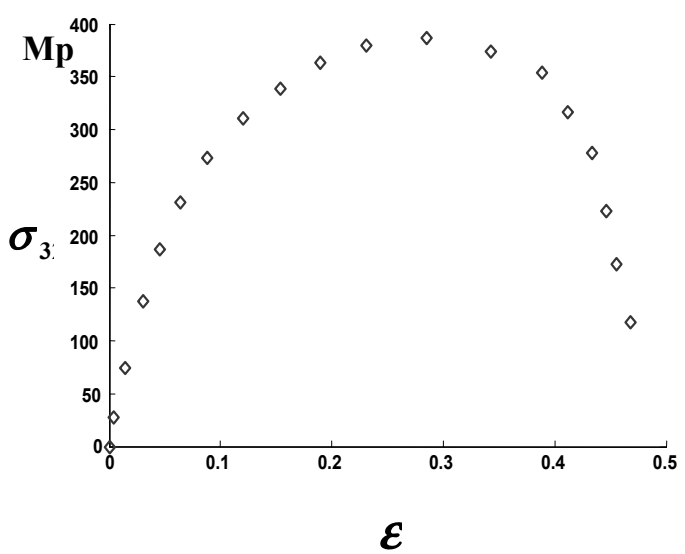

(b)

Fig. 3. (a) Stress versus torsion parameter enhanced finite plasticity model of (MSG) theory for torsion of thin wire (CU), which is implemented in experimental data of fleck et al. (1994) [28]. (b) Stress versus total strain enhenced experimental data for high pressure torsion of nanostructured (CU) Dubravina etal. (Zehetbauer et al., 2002) [29].

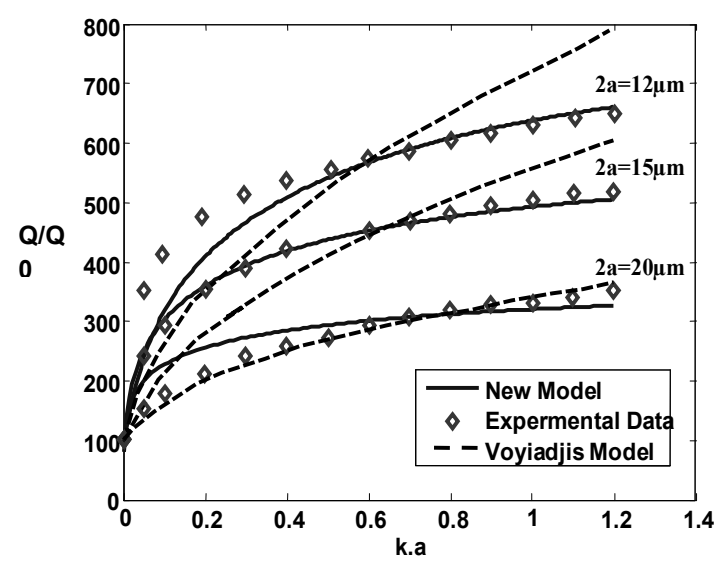

Fig. 4. Comparison of experimental data of Fleck (1994)[28] and Voyadjis model with finite plasticity model of (MSG) for microstructure. 


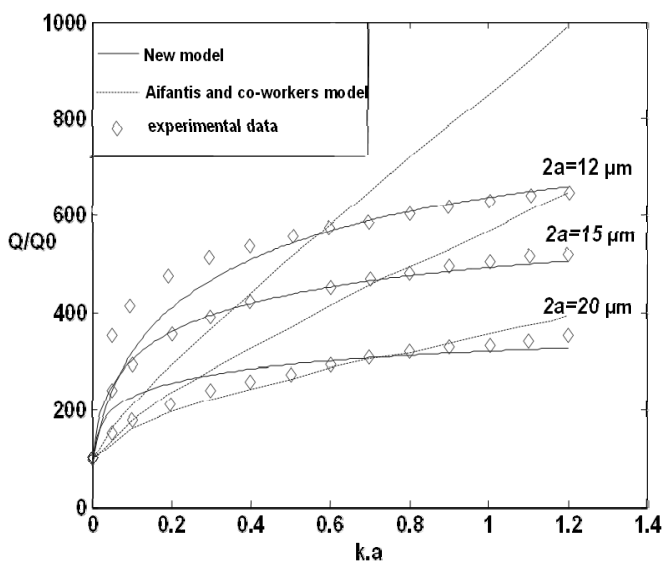

Fig. 5. Comparing new model with experimental data and numerical work by Aifantis and co-workers using second order gradient [23].

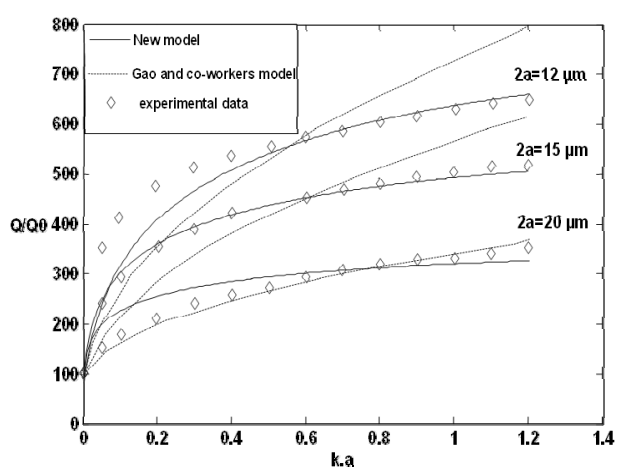

Fig. 6. Comparing new model with experimental data and numerical work by Gao and co-workers using second order gradient [23].

\section{CONCLUSIONS}

In the current work, strain gradient plasticity theory is developed based on the physical properties of phenomena. In this theory, the Taylor's dislocation hardening model and dislocation mechanisms with simple combination GNDs and SSDs in finite plasticity theory, is considering. Involving the dislocation theory as a physically based attitude in finite plasticity form is a novel procedure in generation of equations in classic finite plasticity theory.

Although simple combination between SSDs and GNDs is used in order to bridge the gap between macromechanical and micromechanical plasticity, the implementation of finite plasticity deformation model and suggesting intrinsic length scale relation in micro-torsion test of thin wires, provides results which are significantly fitted to the experimental data.

This model can be predicted the form of stress fluctuation even for nanostructure materials. Furthermore, the better data are extracted from this model when the diameter of microstructure wire is reduced. So, this model may be predicted the acceptable data even for torsion of nanostructure materials and their products.

\section{REFERENCES}

[1] D. J. Bammann and E. C. Aifantis, "On a proposal for a continuum with microstructure," Acta Mech. vol. 45, pp. 91-121, 1982.
[2] A. Arsenlis and D. M. Parks, "Crystallographic aspects of geometrically-necessary and statistically stored dislocation density," Acta Materialia, vol. 47, pp. 1597-1611, 1999.

[3] J. F. Nye, "Some geometrical relations in dislocated crystals," Acta Metallurgica, vol. 1, pp. 153-162, 1953.

[4] M. F Ashby, "The deformation of plastically non-homogeneous materials," Philosophical Magazine, vol. 21, pp. 399-424, 1970.

[5] U. F. Kocks, "A statistical theory of flow stress and work hardening," Phil. Mag. vol. 13, pp. 541, 1966.

[6] U. F. Kocks, "Laws for work-hardening and low-temperature creep," ASME J. Eng. Mater. Tech. vol. 98, pp. 76-85, 1976.

[7] Y. Estrin and H. Mecking, "An unified phenomenological description of work-hardening and creep based on one-parameter models," Acta Metall. vol. 32, pp. 57-70, 1984.

[8] L. P. Kubin and Y. Estrin, "Evolution for dislocation densities and the critical conditions for the portevin-lechatelier effect," Acta Metall. Mater, vol. 38, pp. 697-708, 1990.

[9] D. J. Bammann, "A model of crystal plasticity containing a natural length scale," Mater. Sci. Eng.vol. 309-310 (A), pp. 406-410, 2001.

[10] A. J. Beaudoin and A. Acharya, "A model for rate-dependent flow of metal polycrystals based on the slip plane lattice incompatibility," Mater. Sci. Eng. vol. 309-310 (A), pp. 411-415, 2001.

[11] R. K. Abu Al-Rub and G. Z. Voyiadjis, "Determination of the material intrinsic length scale of gradient plasticity theory," Int. J. Multiscale Comput. Eng. Vol. 2 (b), no.3, pp. 47-70, 2004.

[12] T. Yano, Y. Murakami, D. Shindo, and S. Kuramoto, "Study of the nanostructure of Gum Metal using energy-filtered transmission electron microscopy," Acta Materialia, vol. 57, pp. 628-633, 2009

[13] M. E. Gurtin and L. Annand, "A theory of strain-gradient plasticity for isotropic plastically irrotational materials: II. Finite deformation," Int. J. Plasticity, vol. 21 (b), pp. 2297-2318, 2005

[14] P. Cermelli and M. E. Gurtin, "On the characterization of geometrically necessary dislocation,” J. Mech. Phys. Solid, vol. 49, pp. 1539-1568, 2001.

[15] H. Gao, Y. Huang, W. D. Nix, and J. W. Hutchinson, "Mechanism-based strain gradient plasticity:I," Theory. J. Mech. Phys. Solid, vol. 47, pp. 1239-1036, 1999.

[16] Y. Huang, H. Gao, W. D and J. W. Hutchinson, "Mechanism-based strain gradient plasticity:II," Analysis. J. Mech. Phys. Solid, vol. 48, pp. 99-128, 2000.

[17] R. Chambon, D. Caillerie, and C. Tamagnini, "A strain space gradient plasticity for finite strain," Compu. Methods Appl. Mech. Eng. vol. 193, pp. 2797-2826, 2004.

[18] N. A. Fleck, J. W. Hutchinson, "Strain gradient plasticity," Advances in Applied Mechanics, vol. 33, pp.295-361, 1997.

[19] C. Polizzotto, "A nonlocal strain gradient plasticity theory for finite deformation,” Int. J. Plasticity, vol.25, pp.1280-1300, 2009

[20] A. K Khun and S. Hung, Continuum theory of plasticity: John Wiley \& Sons; 1995

[21] H. Mughrabi, "On the role of strain gradients and long-range internal stresses in the composite model of crystal plasticity," Mater. Sci. Eng. A vol.317, pp.171-180, 2001.

[22] R. K. Abu Al-Rub and G. Z. Voyiadjis, "A physically based gradient plasticity theory," Int. J. plactisity, vol. 22, pp. 654-684, 2005.

[23] R. K. Abu Al-Rub and G. Z. Voyiadjis, "Gradient plasticity theory with a variable length scale parameter," Int. J. solids Struct. vol.42, pp. 3998-4029, 2005.

[24] H. Gao, Y. Huang, and W. D. Nix, "Modeling plasticity at the micrometer scale," Naturwissenschaften, vol.86, pp.507-515, 1999(b).

[25] Y. Huang, H. Gao, W. D. Nix, and J. W. Hutchinson, "Mechanism-based strain gradient plasticity: II," Analysis. J. Mech. Phys. Solid, vol.48, pp. 99-128, 2000.

[26] R. Madec, B. Devincre, and L. P. Kubin, "Simulation of dislocation patterns in multislip," Scripta Materialia, vol.47, pp. 689-695, 2002.

[27] K. C. Hwang, H. Jiang, Y. Huang, and H. Gao, "Finite deformation analysis of mechanism-based strain gradient plasticity: torsion and crack tip field," Int. J. Plasticity, vol. 19, pp. 235-251, 2003.

[28] N. A. Fleck, G. M. Muller, M. F. Ashby and J. W. Hutchinson, "Strain gradient plasticity: theory and experiment," Acta Metall. Mater. vol.42, pp. 475-487, 1994.

[29] A. A. Dubravina, I. V. Alexandrov, R. Z. Valiev, and A.V. Sergueeva, "Deformation behaviour of Copper subjected to high pressure torsion in Zehetbauer," Nano Materials by severe plastic deformation, WILEY-VCH, pp.465-476, 2002. 